\title{
Prevalence and Antimicrobial Resistance of Campylobacter Species in Diarrheal Patients in Mymensingh, Bangladesh
}

\author{
Md. Ashikur Rahman, ${ }^{1}$ Priyanka Rani Paul, ${ }^{1}$ Nazmul Hoque, ${ }^{1}$ Sk Shaheenur Islam ${ }^{D},{ }^{1}$ \\ A. K. M. Ziaul Haque, ${ }^{1}$ Mahmudul Hasan Sikder, ${ }^{2}$ Aminul Matin, ${ }^{3}$ Shinji Yamasaki, \\ and S. M. Lutful Kabir $\left.{ }^{1}\right)^{1}$ \\ ${ }^{1}$ Department of Microbiology and Hygiene, Bangladesh Agricultural University, Mymensingh 2202, Bangladesh \\ ${ }^{2}$ Department of Pharmacology, Bangladesh Agricultural University, Mymensingh 2202, Bangladesh \\ ${ }^{3}$ Health Care Center, Bangladesh Agricultural University, Mymensingh 2202, Bangladesh \\ ${ }^{4}$ Graduate School of Life and Environmental Sciences, Osaka Prefecture University, Osaka 598-8531, Japan
}

Correspondence should be addressed to S. M. Lutful Kabir; lkabir79@bau.edu.bd

Received 19 June 2021; Accepted 20 July 2021; Published 4 August 2021

Academic Editor: Khalid Mehmood

Copyright (c) $2021 \mathrm{Md}$. Ashikur Rahman et al. This is an open access article distributed under the Creative Commons Attribution License, which permits unrestricted use, distribution, and reproduction in any medium, provided the original work is properly cited.

\begin{abstract}
Campylobacter enteritis is the leading cause of gastroenteritis in humans worldwide including Bangladesh. The objectives of this study were to estimate the prevalence and antimicrobial-resistance status of Campylobacter spp. in human diarrheal samples collected from Surya Kanta Hospital, Mymensingh, Bangladesh. In this study, we evaluated a total of 330 clinical samples for the presence Campylobacter spp. via cultural and biochemical tests and molecular assays. Furthermore, antimicrobial susceptibility testing for Campylobacter species was accomplished by the standard agar disc diffusion technique against eight commercially available antimicrobial agents. A pretested semistructured questionnaire was used to capture the data on socioanthropological factors from the diarrheal patients. Pearson's chi-square test was performed, and a $p$ value of $<0.05$ was considered for the level of significance. Nearly one in three diarrheal patients admitted in this hospital were infected with Campylobacter spp. Overall prevalence of Campylobacter spp. was estimated to be 31.5\% (104/330) that comprised the prevalence of $C$. jejuni, $21.8 \%$ ( $n=72$ ), and C. coli, $9.6 \%(n=32)$. Among the positive cases, the prevalence of Campylobacter was higher in the age group 0-5 years (52\%) followed by 6-18 years (42.7\%), 19-40 years (34.0\%), 41-60 years (25.4\%), and >60 years (10.5\%). Age, family level's personal hygiene, and involvement with animal husbandry were captured as potential determinants to be associated with the Campylobacter positive status. Among the isolates, $27.3 \%(n=20)$ of $C$. jejuni and $31.2 \%(n=10)$ of $C$. coli demonstrated as multidrug-resistant (MDR) to three or more antimicrobial agents. The present study shows that Campylobacter spp. is most prevalent among the hospital-admitted diarrheal patients, and proper measures should be taken to reduce the burden focusing on the potential determinants.
\end{abstract}

\section{Introduction}

Campylobacter spp. are considered to be zoonotic pathogens that cause foodborne infection throughout the world $[1,2]$. These pathogens are the leading causative agents of sporadic bacterial diarrhea worldwide $[3,4]$. It was estimated that more than 16 million people got sick with nearly 37 thousand deaths in 2010 worldwide that were connected with Campylobacter spp. as a single infection [3]. Campylobacters are the most frequently isolated bacterial enteric pathogens both in developed and low- and middle-income countries (LMICs) [5]. In developed countries, Campylobacter jejuni and Campylobacter coli are the predominately isolated species associated with human bacterial diarrheal disease [6, 7]. Primarily, most of the zoonotic Campylobacters are well adapted as a commensal in the gastrointestinal tract of poultry, cattle, sheep, and pigs [8] and can act as reservoirs [9]. Humans can be infected with Campylobacter via consumption of contaminated meat and poultry including dairy products $[10,11]$. Additionally, Campylobacter can contaminate 
the soil or water bodies to facilitate human infection or even direct human-to-human transmission [12].

Campylobacter infections exhibit variable clinical signs from sudden diarrhea to vomition, and abdominal cramps with onset of fever could continue at least for a week [13]. Campylobacter jejuni could cause severe chronic symptoms called Guillain-Barré syndrome (GBS) following the infection as a consequence of autoimmune disease [14].

C. jejuni is an important etiological agent of childhood diarrhea (25.5\%) in Bangladesh [15]. However, the Guillain-Barré syndrome (GBS) which is connected to Campylobacter infection causes acute flaccid paralysis (AFP) established in Bangladesh with an estimated incidence rate of 3.25 cases/100,000 children under 15 years of age [16, 17]. Many efforts have been made to minimize Campylobacter infection and its associated GBS risks without considering the source of introduction in the LMICs. Therefore, a significant burden of Campylobacter prevails in such counties [18], even in Bangladesh.

Earlier studies in Bangladesh reported a variable level of prevalence rate during the 1990s ranging from 17 to $26 \%$ with C. jejuni $[19,20]$ and $9.45 \%$ with C. jejuni and $2.68 \%$ with $C$. coli spanning from 2005 to 2008 [21]. A few separate studies confirmed $8.5 \%$ prevalence for Campylobacter jejuni and Campylobacter coli in fecal specimens [22] and $15.3 \%$ and $11.3 \%$ prevalence of Campylobacter jejuni and Campylobacter coli, respectively [23]. However, a most recent study confirmed the prevalence of C. jejuni/coli as $28.3 \%$ in 2019 [24]. Nowadays, the widespread use of antimicrobial agents both in human and veterinary practices is a global concern [25]. This practice appears to be increased quickly in LMICs where rampant use of antibiotics is more common [26]. As a result, the resistance of Campylobacter species to antimicrobials has been documented throughout the world [27]. The emergence of antimicrobial-resistant Campylobacter species due to the overuse of antimicrobial agents in food animal production enables the spreading of antimicrobial-resistant bacteria [28]. This phenomenon highlights a serious impact on both veterinary and human health regarding food safety and public health issues.

In Bangladesh, several studies have been conducted with very few explored socioanthropological determinants of the occurrence of Campylobacter spp. along with antimicrobialresistant pattern of the isolates from human diarrheal patients. Therefore, the present study was designed to estimate the prevalence and antimicrobial resistance profile along with socioanthropological determinants of Campylobacter spp. infection in diarrheal patients from the Surya Kanta Hospital of Mymensingh district in Bangladesh.

\section{Materials and Methods}

2.1. Sample Collection and Shipment. Three hundred thirty $(N=330)$ stool specimens were collected randomly from patients suffering from different magnitudes of acute gastroenteritis symptoms including diarrhea admitted to the Surya Kanta Hospital, Mymensingh Medical College, Mymensingh, during the period from June 2019 to June 2020. A team composed of a physician and a lab technician were engaged in sampling and subsequent data collection. Aseptic measures were taken during the collection of samples. A single rectal swab sample was obtained from each patient, stored, and transported in Cary-Blair transport media. A unique identification number was given for each sample and transferred to the laboratory of the Department of Microbiology and Hygiene, Bangladesh Agricultural University (BAU), Mymensingh, maintaining with a cool chain $\left(4-6^{\circ} \mathrm{C}\right)$.

2.2. Questionnaire Survey. A semistructured interview questionnaire was developed and used for data collection from diarrheal patients on socioanthropological factors: age, sex, educational status, family level personal hygiene, and animal (ruminant, pets, and poultry) husbandry status including food habits. The questionnaire was translated into the local Bengali dialect for the face-to-face interview so that the respondent could easily understand its content.

2.3. Isolation and Identification. Isolation and identification of Campylobacter spp. were accomplished through the filtration method [29]. In brief, each of the stool specimens was suspended in $500 \mu \mathrm{l}$ of sterile saline. A portion of $100 \mu \mathrm{l}$ of sample was spread onto the surface filters of blood agar base no. 2 including Skirrow supplements and permitted to stand for $30 \mathrm{~min}$ at room temperature. After $30 \mathrm{~min}$, the filter was removed from the Skirrow blood agar and then the plates were incubated at $37^{\circ} \mathrm{C}$ for $48 \mathrm{~h}$ in microaerophilic condition $\left(5 \% \mathrm{O}_{2}, 10 \% \mathrm{CO}_{2}\right.$, and $\left.85 \% \mathrm{~N}_{2}\right)$. The incubated media were inspected for bacteria growth after $48 \mathrm{~h}$. In Skirrow blood agar media, grey, flat, and irregularly spreading colonies were observed. The colonies were evaluated via Gram's staining, and Gram-negative curves were observed under the light microscope. Gram's stain positive colonies were further utilized in biochemical tests, namely, catalase, hippurate hydrolysis, and oxidase tests. The selected colonies that were found to be positive in Gram's staining and biochemical tests were further subcultured through the same procedure to get a pure colony. Thus, pure isolates obtained were used for further evaluations. Discrimination of Campylobacter isolates was completed based on growth characteristics as well as biochemical tests as per the standard protocols [30-32].

2.4. Molecular Identification by PCR. Gram's staining and biochemical test positive isolates of the Campylobacter growth colony were validated by PCR assay as Campylobacter spp. The DNA materials were extracted from the pure colonies as per the procedure by Hoshino et al. [33]. Employing oligonucleotide primers, the genus of Campylobacter was confirmed through the amplification of the targeted $16 \mathrm{~S}$ rRNA gene, as per the method labeled by Samosornsuk et al. [34] (Table 1).

After confirmation of Campylobacter spp. via 16S rRNA gene-based PCR assay, a $c d t A$ gene-based multiplex PCR was done for the detection of different species of Campylobacter (i.e., C. jejuni, C. coli, and C. fetus) as per the protocol described by Linton et al. [36]. In these multiplex PCR assays, C. jejuni ATCC 33560, C. coli ATCC 33559, and C. fetus ATCC 27374 strains in DNA templates were utilized as a positive control. However, Escherichia coli ATCC 25922 
TABle 1: Primers and conditions used for different PCR assays.

\begin{tabular}{|c|c|c|c|c|c|c|c|}
\hline \multirow{2}{*}{ Primers } & \multirow{2}{*}{ Sequence $\left(5^{\prime}-3^{\prime}\right)$} & \multirow{2}{*}{ Target/purpose } & \multirow{2}{*}{$\begin{array}{l}\text { Amplicon } \\
\text { size (bp) }\end{array}$} & \multicolumn{3}{|c|}{ PCR condition (30 cycle) } & \multirow{2}{*}{ Reference } \\
\hline & & & & Denaturation & Annealing & Extension & \\
\hline $\begin{array}{l}16 \mathrm{~S} 9 \mathrm{~F} \\
16 \mathrm{~S} 1540 \mathrm{R}\end{array}$ & $\begin{array}{l}\text { GAGTTTGATCCTGGCTC } \\
\text { AAGGAGGTGATCCAGCC }\end{array}$ & 16S rRNA & 1530 & $94^{\circ} \mathrm{C}, 30 \mathrm{~s}$ & $47^{\circ} \mathrm{C}, 30 \mathrm{~s}$ & $72^{\circ} \mathrm{C}, 90 \mathrm{~s}$ & [34] \\
\hline $\begin{array}{l}\text { Cj-CdtAU2 } \\
\text { Cj-CdtAR2 }\end{array}$ & $\begin{array}{l}\text { AGGACTTGAACCTACTTTTC } \\
\text { AGGTGGAGTAGTTAAAAACC }\end{array}$ & $C j c d t A$ & 631 & & & & \\
\hline $\begin{array}{l}\text { Cc-CdtAU1 } \\
\text { Cc-CdtAR1 }\end{array}$ & $\begin{array}{l}\text { ATTGCCAAGGCTAAAATCTC } \\
\text { GATAAAGTCTCCAAAACTGC }\end{array}$ & $C c c d t A$ & 329 & $94^{\circ} \mathrm{C}, 30 \mathrm{~s}$ & $53^{\circ} \mathrm{C}, 30 \mathrm{~s}$ & $72^{\circ} \mathrm{C}, 30 \mathrm{~s}$ & [35] \\
\hline $\begin{array}{l}\text { Cf-CdtCU2 } \\
\text { Cf-CdtCR2 }\end{array}$ & $\begin{array}{l}\text { AACGACAAATGTAAGCACTC } \\
\text { TATTTATGCAAGTCGTGCGA }\end{array}$ & $C f c d t A$ & 489 & & & & \\
\hline $\begin{array}{l}\text { HIP400F } \\
\text { HIP1134R }\end{array}$ & $\begin{array}{l}\text { GAAGAGGGTTTGGGTGGTG } \\
\text { AGCTAGCTTCGCATAATAACTTG }\end{array}$ & hipO gene & 735 & $94^{\circ} \mathrm{C}, 30 \mathrm{~s}$ & $55^{\circ} \mathrm{C}, 30 \mathrm{~s}$ & $72^{\circ} \mathrm{C}, 45 \mathrm{~s}$ & [36] \\
\hline
\end{tabular}

was utilized as a negative control. In the multiplex PCR assay, for those isolates confirmed as $C$. jejuni, their identity was further substantiated by a marker-based (hipO gene) PCR assay [36]. The sequences of the primers and parallel amplicon sizes including annealing temperatures of PCR are shown in Table 1. The PCR products were pictured in gel electrophoresis (1.5\% agarose, Invitrogen, Carlsbad, CA, USA), and after coloring with ethidium bromide $\left(0.5 \mu \mathrm{g} \mathrm{ml}{ }^{-}\right.$ $\left.{ }^{1}\right)$ and decoloring with distilled water for $10 \mathrm{~min}$, gel pictures were captured via a UV transilluminator (Biometra, Göttingen, Germany).

2.5. Antimicrobial Sensitivity Testing. Isolates of Campylobacter spp. were tested via the disk diffusion method [37] using eight (8) commercially available antimicrobials in Bangladesh, viz., amoxicillin $(30 \mu \mathrm{g})$, ciprofloxacin $(5 \mu \mathrm{g})$, azithromycin $(30 \mu \mathrm{g})$, erythromycin $(30 \mu \mathrm{g})$, tetracycline $(30 \mu \mathrm{g})$, streptomycin $(10 \mu \mathrm{g})$, gentamicin $(10 \mu \mathrm{g})$, and ceftriaxone $(30 \mu \mathrm{g})$ (HiMedia, Mumbai, India). The zones of inhibition growth were evaluated as the diameter zone as per parameters specified by the Clinical and Laboratory Standard Institute (CLSI) [38], thus established as resistant (R), intermediate resistant (I) or susceptible (S) against the antimicrobial agents. In this testing, the E. coli ATCC 25922 strain was used as a quality control organism. All evaluations were validated by conducting at least two replications of the disk diffusion test. Multidrug resistance (MDR) was decided as the resistance to a minimum of three different classes of antimicrobial agents [39].

2.6. Data Management and Statistical Analysis. Data on socioanthropological factors and laboratory assessment were captured in Microsoft Excel 2010 (MS Excel) sheets, and data were cleaned and checked for consistency for analysis. The data were analyzed by the Epi Info 7 program [40] both for descriptive and inferential interpretations. Pearson's chisquare test was done to find out the association between determinates and Campylobacter infection status with a $p$ value of $<0.05$ taken as statistical significance for all analyses.

2.7. Ethics Approval and Consent to Participate. The Ethical Committee of the Bangladesh Agricultural University (BAU) approved this study as a part of the larger project
(AWEEC/BAU/2019 (45)). However, a separate permission was obtained from the Director of Mymensingh Medical College Hospital, Mymensingh, Bangladesh (No: 2313 on 8 April 2019) for human screening. Additionally, an oral consent was obtained from each participant as a substantial number of patients included under this study could not read and write.

\section{Results}

3.1. Prevalence of Campylobacter spp. Of 330 samples, 104 were confirmed as Campylobacter spp. via culture, biochemical tests, and, finally, molecular assay (16S rRNA). All positive Campylobacter isolates (104) exhibited a particular amplicon size of $1530 \mathrm{bp}$ through a $16 \mathrm{~S}$ rRNA genespecific polymerase chain reaction (PCR) (Supplementary Figure S1-a). Subsequently, a $c d t A$ gene-based multiplex PCR was accomplished for the detection of different genus of Campylobacter, viz., C. jejuni, C. coli, and C. fetus. In this PCR assay, $C$. jejuni and $C$. coli produced an amplicon size of $631 \mathrm{bp}$ and $329 \mathrm{bp}$, respectively, as a verifying test for species confirmation (Supplementary Figure S1-b). However, the isolates of $C$. jejuni were further confirmed via a robust marker hipO gene-based PCR assay that presented a $735 \mathrm{bp}$ amplicon size (Supplementary Figure S1-c). Thus, the overall prevalence of Campylobacter was confirmed as $31.5 \%(104 / 330)$ that represented $21.8 \%(72 / 330)$ and $9.7 \%$ (32/330) prevalence for C. jejuni and C. coli, respectively (Table 2). In this study, amongst 104 isolates, $69.2 \%$ and $30.8 \%$ were confirmed as C. jejuni and C. coli, respectively.

3.2. Distribution of Prevalence among Different Determinants. A higher prevalence was observed in female (35.2\%) patients than in male (29.3\%) patients. However, there is no statistical significance among the sexes. Considering the age group, the higher prevalence (52\%) was observed in the age group 0-5 years, followed by 6-18 years (42.7\%), 19-40 years (34.0\%), 41-60 years $(25.4 \%)$, and $>60$ years $(10.5 \%)$ (Figure 1 and Table 3).

A lower level of Campylobacter prevalence $(21.5 \%)$ was documented in good hygienic practice patients and found to be statistically significant. Similarly, involvement of livestock (cattle, sheep, goat, and poultry) rearing was found to 
TABLE 2: Distribution of Campylobacter isolates in human diarrheal samples $(N=330)$ at Surya Kanta Hospital, Mymensingh, from June 2019 to June 2020.

\begin{tabular}{lcc}
\hline Parameter/variable & $\begin{array}{c}\text { Positive sample } \\
(n) / \text { number of } \\
\text { sample tested }(N)\end{array}$ & $\begin{array}{c}\text { Prevalence (\%) } \\
\text { with 95\% CI }\end{array}$ \\
\hline Campylobacter spp. & $104 / 330$ & $31.5(26.5-36.8)$ \\
Campylobacter jejuni $(n=72)$ & $72 / 330$ & $21.8(17.5-26.7)$ \\
Campylobacter coli $(n=32)$ & $32 / 330$ & $9.7(6.7-13.4)$ \\
\hline
\end{tabular}

$N$ : total sample tested; $n$ : number of positive isolates; CI: confidence interval.

be risky practices as higher prevalence $(43.2 \%)$ was recognized. Similarly, regarding food habits, Campylobacter was found to be more prevalent in those patients who ate home-prepared food than the patients who ate other sources of food. However, no statistical significance was found (Table 2).

\subsection{Antibiogram}

3.3.1. Antimicrobial Susceptibility Testing. In this study, of 72 isolates of $C$. jejuni, $54.2 \%(n=39)$ were found to be susceptible to azithromycin, followed by streptomycin $(51.4 \%, n$ $=37)$, gentamycin and/or ceftriaxone $(47.2 \%, n=34)$, ciprofloxacin $(44.4 \%, n=32)$, tetracycline $(40.3 \%, n=29)$, erythromycin $(33.4 \%, n=24)$, and amoxicillin $(20.8 \%, n=15)$. However, $34.7 \%(n=25)$ isolates exhibited intermediate susceptibility to gentamycin and/or streptomycin and 30.6\% $(n=22)$ isolates to azithromycin and/or ceftriaxone. On the contrary, 32 isolates of C. coli $62.5 \%(n=20)$ were found susceptible to ceftriaxone and/or ciprofloxacin and streptomycin followed by gentamycin $(37.5 \%, n=12)$, azithromycin $(28.1 \%, n=9)$, tetracycline $(21.9 \%, n=7)$, and amoxicillin $(18.8 \%, n=6)$. Conversely, $40.6 \%(n=13)$ isolates were shown intermediately susceptible to azithromycin followed by streptomycin $(28.1 \%, n=9)$ and tetracycline $(18.8 \%, n=6)$ (Table 4).

3.3.2. Antimicrobial-Resistant Status. Among the 72 isolates of C. jejuni, $72.3 \%(n=52)$ were presented as resistant against 1-2 antimicrobial agents that comprised 33.4\% $(n=24)$ isolates to single antimicrobial agents (AMX, ERY) and $38.9 \%(n=28)$ to two antimicrobial agents (AMX-TET and AMX-STR). Similarly, of 32 isolates of C. coli, $68.7 \%$ $(n=22)$ were presented as resistant against 1-2 antimicrobial agents that comprised $50.0 \%(n=16)$ isolates to single antimicrobial agents (AMX, ERY) and $18.7 \%(n=6)$ to two antimicrobial agents (AMX-TET and AMX-STR).

In this study, among the isolates of $C$. jejuni $(N=72), 10$ (13.9\%), $2(2.8 \%)$, and 4 (5.6\%) were found to be multidrugresistant against three antimicrobial agents, namely, AMXSTR-TET, ERY-STR-CIP, and AMX-TET-CRO, respectively. However, 4 (5.56\%) isolates were documented to be resistant against 4 antimicrobial agents (AMX-TET-CROGEN) (Figure 2(a)).

Correspondingly, among the isolates of $C$. coli $(N=32), 2$ (6.2\%), $4(12.4 \%)$, and $2(6.2 \%)$ were found to be multidrug- resistant against three antimicrobial agents, viz., AMX-STRTET, ERY-STR-CIP, and AMX-TET-ERY, respectively. Nevertheless, 2 (6.3\%) isolates were shown resistant against 4 antimicrobial agents (AMX-TET-CRO-GEN) in this study (Figure 2(b)).

\section{Discussion}

We evaluated the prevalence, risk factors, and antimicrobial resistant status of Campylobacter spp. in diarrheal specimens collected from the patients admitted in Surya Kanta Hospital, Mymensingh, Bangladesh. The study confirmed that nearly one-third of the patients (31.5\%) was found to be positive with Campylobacter spp., of which Campylobacter jejuni (21.8\%) was captured as the key contributor of human bacterial diarrhea. The finding of this study is in accordance to similar studies conducted in Bangladesh $[15,19-23]$.

In this study, the prevalence of Campylobacter spp. was found higher in females than the males but not statistically significant. In Bangladesh, females are more likely to be at an increased risk of Campylobacter exposure as they are involved with household livestock rearing (cattle, sheep, goat, and poultry) than males. This finding conforms to the result of an earlier study conducted in Bangladesh [23]. However, improved personal hygiene practices would contribute towards the lower prevalence in males.

The distribution of prevalence of Campylobacter spp. was found to be significantly varied among the different age groups $(p \leq 0.001)$. In this study, the higher prevalence was observed in the youngest age group ( $0-5$ years). Earlier studies also reported variable prevalence of Campylobacter as $25.5 \%$ [15] and $12.9 \%$ [41] but consistent with the most susceptible young patients. Campylobacter can cause infection in all age groups; however, the clinical presentation may differ by different age groups of patients established in previous studies [23, 42-44]. Children at a young age are not aware on hygiene and sanitation practices. Commonly, in the rural settings of Bangladesh, farmers keep the livestock close to their house which offers regular contact of young children with the animals which is likely to further the risk of exposure of infection.

Family-level personal hygiene and involvement with livestock rearing were found to be associated with a higher level of Campylobacter exposure. The main routes of transmission of Campylobacter are the consumption of poultry and involvement with poultry rearing activities [45] or exposures resulting from environmental contamination by cattle manure $[46,47]$ that facilitate potential introduction in humans through the food chain [48]. However, exposure to livestock was documented as a significant association with higher Campylobacter positivity status [49]. Therefore, family-level involvement with livestock rearing including poor personal hygiene was presented to be a higher level of Campylobacter occurrence in this study.

Our study found that 20.8 to $51.4 \%$ and 15.6 to $62.5 \%$ isolates of C. jejuni $(n=72)$ and C. coli $(n=30)$, respectively, were susceptible to all antimicrobial agents. The isolates $C$. jejuni and C. coli exhibited resistant to amoxicillin 


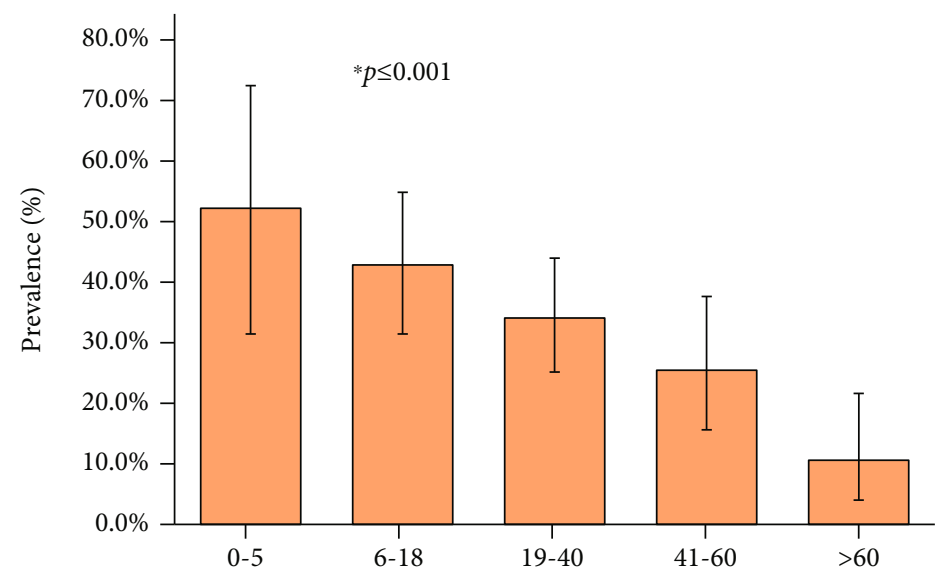

Figure 1: Distribution of prevalence with 95\% confidence interval (CI) at different age groups in Surya Kanta Hospital, Mymensingh, from June 2019 to June 2020 . The prevalence among the different age groups was found to be statistically significant from each other $\left({ }^{*} p \leq 0.001\right)$.

Table 3: Distribution of Campylobacter spp. among different determinants of diarrheal patients $(N=330)$ at Surya Kanta Hospital, Mymensingh, from June 2019 to June 2020.

\begin{tabular}{|c|c|c|c|}
\hline Determinants $(n)$ & Positive & Prevalence (\%) with 95\% CI & Pearson's chi-square $p$ value \\
\hline \multicolumn{4}{|l|}{ Sex } \\
\hline Male $(n=208)$ & 61 & $29.3(23.2-26.0)$ & \multirow{2}{*}{0.26} \\
\hline Female $(n=122)$ & 43 & $35.3(26.8-44.4)$ & \\
\hline \multicolumn{4}{|l|}{ Age (years) } \\
\hline $0-5(n=25)$ & 13 & $52.0(31.3-72.2)$ & \multirow{5}{*}{$\leq 0.001$} \\
\hline $6-18(n=75)$ & 32 & $42.7(31.3-54.7)$ & \\
\hline $19-40(n=106)$ & 36 & $34.0(25.0-43.8)$ & \\
\hline $41-60(n=67)$ & 17 & $25.4(15.5-37.5)$ & \\
\hline$>60(n=57)$ & 6 & $10.5(4.0-21.5)$ & \\
\hline \multicolumn{4}{|l|}{ Education status } \\
\hline Illiterate/not applicable & 56 & $37.7(26.6-41.5)$ & \multirow{2}{*}{0.38} \\
\hline Literate & 48 & $29.3(22.4-36.9)$ & \\
\hline \multicolumn{4}{|l|}{ Family-level personal hygiene } \\
\hline Good $(n=228)$ & 49 & $21.5(16.3-27.4)$ & \multirow{2}{*}{$\leq 0.001$} \\
\hline $\operatorname{Bad}(n=102)$ & 55 & $53.9(43.8-63.8)$ & \\
\hline \multicolumn{4}{|l|}{ Involvement with household livestock rearing } \\
\hline Yes $(n=132)$ & 57 & $43.2(34.6-52.1)$ & \multirow{2}{*}{0.001} \\
\hline No $(n=198)$ & 47 & $23.7(18.0-30.3)$ & \\
\hline \multicolumn{4}{|l|}{ Food habit } \\
\hline Home prepared $(n=227)$ & 77 & $33.9(27.8-40.5)$ & \multirow{2}{*}{0.16} \\
\hline Other (fast food/food from restaurant) $(n=103)$ & 27 & $26.2(18.0-35.8)$ & \\
\hline
\end{tabular}

CI: confidence interval.

(54.2\%), erythromycin (45.8\%), tetracycline (43\%), ciprofloxacin $(33.3 \%)$, and azithromycin $(22.2 \%)$ and amoxicillin $(68.7 \%)$, erythromycin $(62.5 \%)$, tetracycline $(59.3 \%)$, and ciprofloxacin and/or azithromycin (31.5\%), respectively. This finding is narrowly corroborated by Albert et al. [19] in Bangladesh as Campylobacter isolates show resistance to ciprofloxacin that varies from 65 to $88 \%$ during 2005 to 2008 [21]. However, the finding of this study is consistent with a similar study conducted in diarrheal patients in Fin- land [50]. However, antimicrobial resistance was documented as $46.7 \%, 35.6 \%$, and $17.8 \%$ in C. jejuni, C. coli, and C. upsaliensis isolates, respectively, in beef cattle of South Africa [51]. Conversely, higher levels of resistance to ciprofloxacin and tetracycline as $>80 \%$ and $>70 \%$, respectively, were captured in the diarrheal sample in the Arabian Gulf region [52].

Our study demonstrated that $27.8 \%(n=20)$ and $31.2 \%$ $(n=10)$ of $C$. jejuni and $C$. coli, respectively, showed as 
TABLE 4: Antimicrobial susceptibility pattern of Campylobacter jejuni $(n=72)$ and Campylobacter coli $(n=32)$ identified by the disk diffusion method at Surya Kanta Hospital, Mymensingh, from June 2019 to June 2020.

\begin{tabular}{|c|c|c|c|c|c|c|}
\hline \multirow[t]{2}{*}{ Antimicrobial agents } & \multicolumn{2}{|c|}{$\begin{array}{l}\text { Susceptible }(\%, n) \text { rate of } \\
\text { isolates by species }\end{array}$} & \multicolumn{2}{|c|}{$\begin{array}{l}\text { Intermediate }(\%, n) \text { rate of } \\
\text { isolates by species }\end{array}$} & \multicolumn{2}{|c|}{$\begin{array}{l}\text { Resistant }(\%, n) \text { rate of } \\
\text { isolates by species }\end{array}$} \\
\hline & C. jejuni & C. coli & C. jejuni & C. coli & C. jejuni & C. coli \\
\hline Amoxicillin (AMX) & $20.8(15)$ & $18.8(6)$ & $25(18)$ & $12.5(4)$ & $54.2(39)$ & $68.7(22)$ \\
\hline Tetracycline (TET) & $40.3(29)$ & $21.9(7)$ & $16.7(12)$ & $18.8(6)$ & $43(31)$ & $59.3(19)$ \\
\hline Gentamicin (GEN) & $47.2(34)$ & $37.5(12)$ & $34.7(25)$ & $12.5(4)$ & $18.1(13)$ & $50(16)$ \\
\hline Streptomycin (ST) & $51.4(37)$ & $62.5(20)$ & $34.7(25)$ & $28.1(9)$ & $13.9(10)$ & $9.4(3)$ \\
\hline Erythromycin (ERY) & $33.4(24)$ & $15.6(5)$ & $20.8(15)$ & $21.9(7)$ & $45.8(33)$ & $62.5(20)$ \\
\hline Azithromycin (AZM) & $54.2(39)$ & $28.1(9)$ & $30.6(22)$ & $40.6(13)$ & $15.2(11)$ & $31.3(10)$ \\
\hline Ciprofloxacin (CIP) & $44.4(32)$ & $62.5(20)$ & $22.3(16)$ & $6.3(2)$ & $33.3(24)$ & $31.2(10)$ \\
\hline Ceftriaxone (CRO) & $47.2(34)$ & $62.5(20)$ & $30.6(22)$ & $6.3(2)$ & $22.2(16)$ & $31.2(10)$ \\
\hline
\end{tabular}

$n$ : number of isolates; \%: percentage.

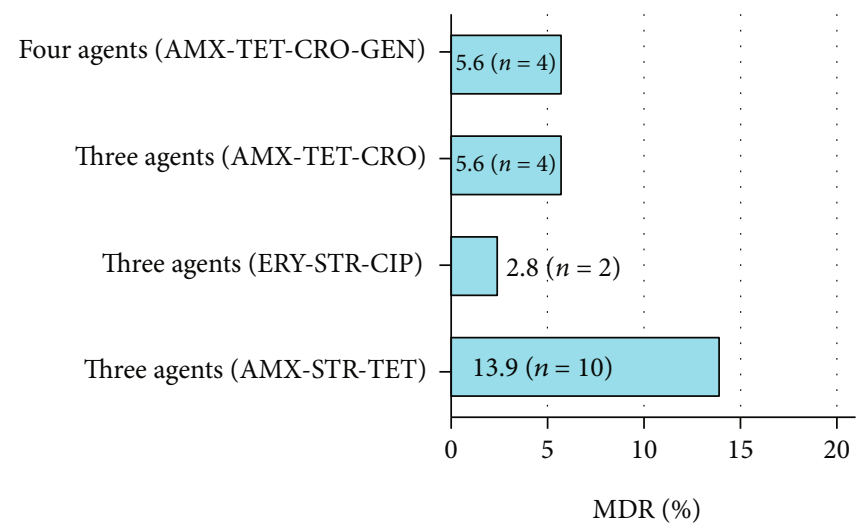

(a)

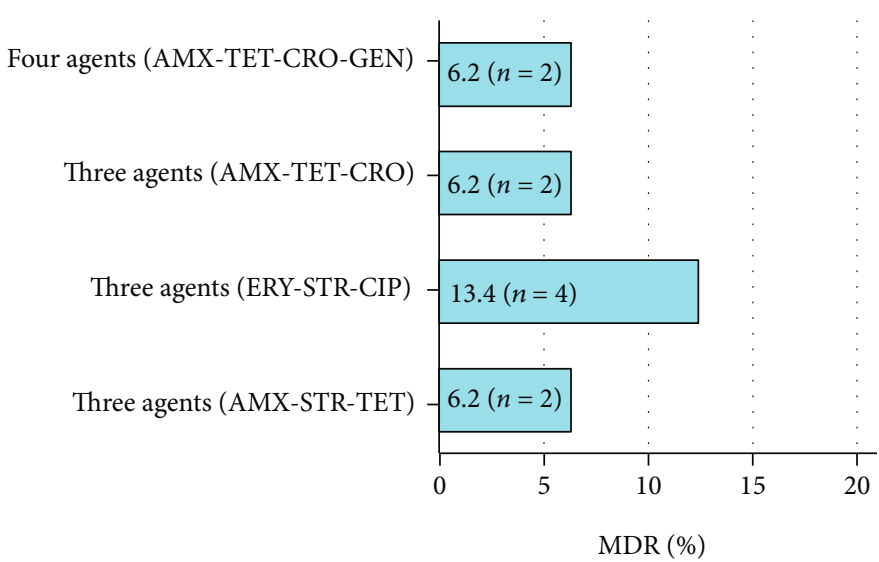

(b)

FIgURE 2: Multidrug-resistant (MDR) status of (a) C. jejuni isolates $(n=72)$ presented resistant to 3 agents (AMX-STR-TET) $(n=10$, $13.9 \%), 3$ agents (ERY-STR-CIP) $(n=2,2.8 \%), 3$ agents (AMX-TET-CRO) $(n=4,5.6 \%)$, and 4 agents (AMX-TET-CRO-GEN) $(n=4$, $5.6 \%)$; (b) C. coli isolates $(n=32)$ presented resistant to 3 agents (AMX-STR-TET) $(n=2,6.2 \%), 3$ agents (ERY-STR-CIP) $(n=4,12.4 \%)$, 3 agent (AMX-TET-CRO) $(n=2,6.2 \%)$, and 4 agents (AMX-TET-CRO-GEN) $(n=2,6.2 \%)$ at Surya Kanta Hospital, Mymensingh, from June 2019 to June 2020 .

multidrug resistance to 3 or more antimicrobial drugs, consistent with similar studies [23, 50, 53-55]. The MDR noticed in this survey is likely to be a potential reflection of imprudent use of antimicrobials due to their easy accessibility at local drug stores throughout the country [21].
Based on the study findings, risk reduction measures to be taken to address health education for the household livestock keepers, personal hygiene, microbiological evaluation of the isolates through culture and molecular assessment, and prudent use antimicrobials are needed. 


\section{Conclusions}

The present study shows occurrence of Campylobacter infection in diarrheal patients, of which Campylobacter jejuni was captured as an abundant species. The higher frequency was observed in 0-5 years age, family-level poor hygienic practices, and involvement of animal husbandry. Therefore, activities on awareness creating behavioral change relating to personal hygiene like hand washing and sanitization after using the toilet or animal contact are needed. Children 0-5 years of age should avoid contact with livestock. The treatment of diarrhea patients should be based on an updated database on the susceptibility status of Campylobacter spp. instead of clinical signs. A physician should consider that diagnosing diarrhea infection with Campylobacter spp. is crucial among the other enteric pathogens. Cognizing the burden of Campylobacter diarrheal disease is significant for framing effective control programs targeting the overall decline of diarrheal disease in all ages of people. Further investigations are required to substantiate the role of domestic animals in the spreading of Campylobacter spp. including species diversities/serotyping.

\section{Data Availability}

The tables, figures and texts in this research article contain the data that support the study conclusions.

\section{Conflicts of Interest}

The authors declare that no conflict of interest exists.

\section{Acknowledgments}

The authors are thankful to the Mymensingh Medical Collage authority for allowing the screening of diarrheal patients under this study. The authors would also like to extend their appreciation to all participants for their cooperation during the sampling and subsequent interview data collection. This research was funded by the Krishi Gobeshona Foundation (Project ID: TF-45-L/17), AIC Building (3rd Floor), BARC Campus, Farmgate, Dhaka-1215, Bangladesh.

\section{Supplementary Materials}

Supplementary Figure S1: molecular detection: (a) confirmation of Campylobacter spp. by the 16S rRNA gene, lanes 1 and 12: $100 \mathrm{bp}$ DNA ladder (Promega, USA), lanes 2-9: representative positive isolates, lane 10: positive control (C. jejuni ATCC 33560), and lane 11: negative control (Escherichia coli ATCC 25922); (b) confirmation of C. jejuni and C. coli via $c d t A$ gene-based multiplex PCR assay, lanes 1 and 12: $100 \mathrm{bp}$ DNA ladder (Promega, USA), lanes 2-4: representative positive isolates (C. coli), lanes 5-7: representative positive isolates (C. jejuni), lane 8: positive control (C. fetus ATCC 27374), lane 9: positive control (C. coli ATCC 33559), lane 10: positive control (C. jejuni ATCC 33560), and lane 11: negative control (Escherichia coli ATCC 25922); (c) validation of $C$. jejuni by hippuricase (hipO) gene-based PCR, lanes 1 and 12: 100 bp DNA lad- der (Promega, USA), lanes 2-9: representative positive isolates, lane 10: positive control (C. jejuni ATCC 33560), and lane 11: negative control (Escherichia coli ATCC 25922). (Supplementary Materials)

\section{References}

[1] L. Zhang, Y. Li, Y. Shao et al., "Molecular characterization and antibiotic resistant profiles of Campylobacter species isolated from poultry and diarrheal patients in Southeastern China 2017-2019," Frontiers Microbiology, vol. 11, no. 1244, 2020.

[2] D. F. Hlashwayo, B. Sigaúque, and C. G. Bila, "Epidemiology and antimicrobial resistance of Campylobacter spp. in animals in Sub-Saharan Africa: A systematic review," Heliyon, vol. 6, no. 3, p. e03537, 2020.

[3] M. D. Kirk, S. M. Pires, R. E. Black et al., "Correction: World Health Organization estimates of the global and regional disease burden of 22 foodborne bacterial, protozoal, and viral diseases, 2010: a data synthesis," PLoS Medicine, vol. 12, no. 12, p. e1001940, 2015.

[4] Y. Li, S. Zhang, M. He et al., "Prevalence and molecular characterization of Campylobacter spp. isolated from patients with diarrhea in Shunyi, Beijing," Frontiers Microbiology, vol. 9, 2018.

[5] S. M. L. Kabir, Comparison of Molecular Methods for the Species Identification of Clinical Campylobacter Strains and Their Antimicrobial Resistance, [Ph.D. thesis], Osaka Prefecture University, Osaka, Japan, 2011.

[6] C. R. Friedman, J. Neimann, H. C. Wegener, and R. V. Tauxe, "Epidemiology of Campylobacter jejuni infections on the United States and other industrialized nations," in Campylobacter, I. Nachamkin and M. J. Blaser, Eds., pp. 121-138, ASM press, Washington DC, USA, 2nd edition, 2000.

[7] European Food Safety Authority and European Centre for Disease Prevention and Control, "The European Union summary report on trends and sources of zoonoses, zoonotic agents and food-borne outbreaks in 2013," EFSA Journal, vol. 13, no. 1, 2015.

[8] O. Sahin, M. Yaeger, Z. Wu, and Q. Zhang, "Campylobacterassociated diseases in animals," Annual Review of Animal Biosciences, vol. 5, no. 1, pp. 21-42, 2017.

[9] M. Mäesaar, T. Tedersoo, K. Meremäe, and M. Roasto, “The source attribution analysis revealed the prevalent role of poultry over cattle and wild birds in human campylobacteriosis cases in the Baltic States," PLoS One, vol. 15, 2020.

[10] S. K. Sheppard, J. F. Dallas, N. J. Strachan et al., "Campylobacter genotyping to determine the source of human infection," Clinical Infectious Disease, vol. 48, no. 8, pp. 10721078, 2009.

[11] W. G. Miller and R. E. Mandrell, "Prevalence of Campylobacter in the food and water supply: incidence, outbreaks, isolation and detection," in Campylobacter jejuni: New Perspectives in Molecular and Cellular Biology, J. Ketley and M. E. Konkel, Eds., pp. 101-163, Horizon Scientific Press, Norfolk, UK, 2005.

[12] M. J. Blaser, "Epidemiologic and clinical features of Campylobacter jejuni Infections," Journal of Infectious Disease, vol. 176, no. s2, pp. S103-S105, 1997.

[13] H. Goossens, L. Vlaes, M. de Boeck et al., "Is "Campylobacter upsaliensis" an unrecognised cause of human diarrhoea?," Lancet, vol. 335, no. 8689, pp. 584-586, 1990.

[14] K. K. Nyati and R. Nyati, "Role of Campylobacter jejuni infection in the pathogenesis of Guillain-Barré syndrome: an 
update," Biomedical Research International, vol. 2013, article 852195, 13 pages, 2013.

[15] J. A. Haq and K. M. Rahman, "Campylobacter jejuni as a cause of acute diarrhoea in children: a study at an urban hospital in Bangladesh," Journal of Tropical Medicine and Hygiene, vol. 94, pp. 50-54, 1991.

[16] Z. Islam, B. C. Jacobs, M. B. Islam, Q. D. Mohammad, S. Diorditsa, and H. P. Endtz, "High incidence of GuillainBarre syndrome in children, Bangladesh," Emerging Infectious Disease, vol. 17, no. 7, pp. 1317-1318, 2011.

[17] Z. Islam, M. Gilbert, Q. D. Mohammad et al., "Guillain-Barré syndrome-related Campylobacter jejuni in Bangladesh: ganglioside mimicry and cross-reactive antibodies," PLoS One, vol. 7, no. 8, p. e43976, 2012.

[18] J. A. Platts-Mills and M. Kosek, "Update on the burden of Campylobacter in developing countries," Current Opinion in Infectious Diseases, vol. 27, no. 5, pp. 444-450, 2014.

[19] M. J. Albert, A. Faruque, S. Faruque, R. B. Sack, and D. Mahalanabis, "Case-control study of enteropathogens associated with childhood diarrhea in Dhaka, Bangladesh," Journal of Clinical Microbiology, vol. 37, no. 11, pp. 34583464, 1999.

[20] S. S. Hoque, A. Faruque, D. Mahalanabis, and A. Hasnat, "Infectious agents causing acute watery diarrhoea in infants and young children in Bangladesh and their public health implications," Journal of Tropical Pediatrics, vol. 40, no. 6, pp. 351-354, 1994.

[21] D. Ahmed, A. Hoque, M. S. B. Elahi, H. P. Endtz, and M. A. Hossain, "Bacterial aetiology of diarrhoeal diseases and antimicrobial resistance in Dhaka, Bangladesh, 2005-2008," Epidemiology \& Infection, vol. 140, no. 9, pp. 1678-1684, 2012.

[22] K. Alam, A. J. Lastovica, E. Le Roux et al., "Clinical characteristics and serotype distribution of Campylobacter jejuni and Campylobacter coli isolated from diarrhoeic patients in Dhaka, Bangladesh, and Cape Town, South Africa," Bangladesh Journal of Microbiology, vol. 23, pp. 121-124, 2008.

[23] S. Karmaker, S. M. L. Kabir, A. Z. Haque, F. R. K. Mohammad, and A. S. Yousuf, "Screening of human diarrhoeal samples in Mymensingh city of Bangladesh for the isolation, identification and antimicrobial resistance profiles of Campylobacter spp," African Journal of Microbiology Research, vol. 12, no. 32, pp. 771-778, 2018.

[24] The PLOS Neglected Tropical Diseases Staff, "Campylobacter infection and household factors are associated with childhood growth in urban Bangladesh: an analysis of the MAL-ED study," PLoS Neglected Tropical Diseases, vol. 15, 2021.

[25] M. M. Hassan, K. B. Amin, M. Ahaduzzaman, M. Alam, M. S. A. Faruk, and I. Uddin, "Antimicrobial resistance pattern against E. coli and Salmonella in layer poultry," Research Journal for Veterinary Practitioners, vol. 2, no. 2, pp. 30-35, 2014.

[26] M. D. Englen, S. R. Ladely, and P. J. Fedorka-Cray, "Isolation of Campylobacter and identification by PCR," Methods in Molecular Biology, vol. 216, pp. 109-221, 2003.

[27] D. W. Isenbarger, C. W. Hoge, A. Srijan et al., "Comparative antibiotic resistance of diarrheal pathogens from Vietnam and Thailand, 1996-1999," Emerging Infectious Disease, vol. 8, no. 2, pp. 175-180, 2002.

[28] J. Engberg, J. Neimann, E. M. Nielsen, F. M. Aarestrup, and V. Fussing, "Quinolone-resistant Campylobacter infections: risk factors and clinical consequences," Emerging Infectious Disease, vol. 10, no. 6, pp. 1056-1063, 2004.
[29] S. Shiramaru, M. Asakura, H. Inoue, A. Nagita, A. Matsuhisa, and S. Yamasaki, "A cytolethal distending toxin gene-based multiplex PCR assay for detection of Campylobacter spp. in stool specimens and comparison with culture method," Veterinary Medicine and Science, vol. 74, no. 7, pp. 857-862, 2012.

[30] I. Nachamkin, "Campylobacter and Arcobacter," in Manual of Clinical Microbiology, pp. 902-914, ASM press, Washington, DC, USA, 8th edition, 2003.

[31] G. Foster, B. Holmes, A. G. Steigerwalt et al., "Campylobacter insulaenigrae sp. nov., isolated from marine mammals," International Journal of Systematic and Evolutionary Microbiology, vol. 54, no. 6, pp. 2369-2373, 2004.

[32] E. Swai, J. Hulsebosch, and W. van der Heijden, "Prevalence of genital campylobacteriosis and trichomonosis in crossbred breeding bulls kept on zero-grazed smallholder dairy farms in the Tanga region of Tanzania," Journal of the South African Veterinary Association, vol. 76, no. 4, pp. 224-227, 2005.

[33] K. Hoshino, S. Yamasaki, A. K. Mukhopadhyay et al., "Development and evaluation of a multiplex PCR assay for rapid detection of toxigenic Vibrio cholerae O1 and O139," FEMS Immunology Medical Microbiology, vol. 20, no. 3, pp. 201207, 1998.

[34] W. Samosornsuk, M. Asakura, E. Yoshida et al., "Evaluation of a cytolethal distending toxin $(c d t)$ gene-based species-specific multiplex PCR assay for the identification of Campylobacter strains isolated from poultry in Thailand," Microbiology \& Immunology, vol. 51, no. 9, pp. 909-917, 2007.

[35] M. Asakura, W. Samosornsuk, A. Hinenoya et al., "Development of a cytolethal distending toxin $(c d t)$ gene-based species-specific multiplex PCR assay for the detection and identification of Campylobacter jejuni, Campylobacter coli and Campylobacter fetus," FEMS Immunology and Medical Microbiology, vol. 52, no. 2, pp. 260-266, 2008.

[36] D. Linton, A. Lawson, R. Owen, and J. Stanley, "PCR detection, identification to species level, and fingerprinting of Campylobacter jejuni and Campylobacter coli direct from diarrheic samples," Journal of Clinical Microbiology, vol. 35, no. 10, pp. 2568-2572, 1997.

[37] T. Luangtongkum, T. Y. Morishita, A. B. el-Tayeb, A. J. Ison, and Q. Zhang, "Comparison of antimicrobial susceptibility testing of Campylobacter spp. by the agar dilution and the agar disk diffusion methods," Journal of Clinical Microbiology, vol. 45, no. 2, pp. 590-594, 2007.

[38] Clinical and Laboratory Standards Institute (CLSI), M100 Performance standards for antimicrobial susceptibility testing, CLSI supplement, Wayne, PA, USA, 26th edition, 2016.

[39] A. P. Magiorakos, A. Srinivasan, R. B. Carey et al., "Multidrugresistant, extensively drug-resistant and pandrug-resistant bacteria: an international expert proposal for interim standard definitions for acquired resistance," Clinical Microbiology and Infection, vol. 18, no. 3, pp. 268-281, 2012.

[40] Centers for Disease Control and Prevention (CDC), "Epi Info $^{\mathrm{TM}} 7$," in User Guide, pp. 1-645, CDC, 2016.

[41] N. Huda, S. Andalib, and M. A. Yusuf, "Socio-demographic characteristics of Campylobacter jejuni infected diarrhoeal patients under 5 years," Bangladesh Journal of Infectious Diseases, vol. 2, no. 2, pp. 33-36, 2017.

[42] A. O. Coker, R. D. Isokpehi, B. N. Thomas, K. O. Amisu, and C. L. Obi, "Human campylobacteriosis in developing countries," Emerging Infectious Disease, vol. 8, 2007. 
[43] R. A. Oberhelman and D. N. Taylor, "Campylobacter infections in developing countries," in Campylobacter, I. Nachamkin and M. J. Blaser, Eds., pp. 139-153, American Society for Microbiology, Washington, DC, USA, 2nd edition, 2000.

[44] D. Taylor, "Campylobacter infections in developing countries," in Campylobacter jejuni: Current status and future trends, I. Nachamkin, M. J. Blaser, and L. S. Tompkins, Eds., pp. 2030, American Society for Microbiology, Washington, DC, USA, 1992.

[45] J. Arsenault, P. Michel, O. Berke, A. Ravel, and P. Gosselin, "Environmental characteristics associated with campylobacteriosis: accounting for the effect of age and season," Epidemiology \& Infection, vol. 140, no. 2, pp. 311-322, 2012.

[46] C. Manyi-Loh, S. Mamphweli, E. Meyer, G. Makaka, M. Simon, and A. Okoh, "An overview of the control of bacterial pathogens in cattle manure," International Journal of Environmental Research and Public Health, vol. 13, 2016.

[47] E. Mourkas, D. Florez-Cuadrado, B. Pascoe et al., "Gene pool transmission of multidrug resistance among Campylobacter from livestock, sewage and human disease," Environmental Microbiology, vol. 21, no. 12, pp. 4597-4613, 2019.

[48] E. Mourkas, A. J. Taylor, G. Méric et al., "Agricultural intensification and the evolution of host specialism in the enteric pathogen Campylobacter jejuni," Proceedings of the National Academy of Sciences of the United States of America, vol. 117, no. 20, pp. 11018-11028, 2020.

[49] A. Lengerh, F. Moges, C. Unakal, and B. Anagaw, "Prevalence, associated risk factors and antimicrobial susceptibility pattern of Campylobacter species among under five diarrheic children at Gondar University Hospital, Northwest Ethiopia," BMC Pediatric, vol. 13, no. 82, pp. 1-9, 2013.

[50] A. J. Hakanen, M. Lehtopolku, A. Siitonen, P. Huovinen, and P. Kotilainen, "Multidrug resistance in Campylobacter jejuni strains collected from Finnish patients during 1995-2000," Journal of Antimicrobial Chemotherapy, vol. 52, no. 6, pp. 1035-1039, 2003.

[51] M. Karama, K. Kambuyi, B. T. Cenci-Goga et al., "Occurrence and antimicrobial resistance profiles of Campylobacter jejuni, Campylobacter coli, and Campylobacter upsaliensis in beef cattle on cow-calf operations in South Africa," Foodborne Pathogens and Disease, vol. 17, no. 7, pp. 440-446, 2020.

[52] A. Senok, A. Yousif, W. Mazi et al., "Pattern of antibiotic susceptibility in campylobacter jejuni isolates of human and poultry origin," Japanese Journal of Infectious Diseases, vol. 60, no. 1, pp. 1-4, 2007.

[53] K. McGill, D. Cowley, L. Moran et al., "Antibiotic resistance of retail food and human Campylobacter isolates on the island of Ireland from 2001-2002," Epidemiology \& Infection, vol. 134, no. 6, pp. 1282-1291, 2006.

[54] J. E. Moore, M. D. Barton, I. S. Blair et al., "The epidemiology of antibiotic resistance in Campylobacter," Microbes Infection, vol. 8, no. 7, pp. 1955-1966, 2006.

[55] T. Luangtongkum, B. Jeon, J. Han, P. Plummer, C. M. Logue, and Q. Zhang, "Antibiotic resistance in Campylobacter: emergence, transmission and persistence," Future Microbiology, vol. 4, no. 2, pp. 189-200, 2009. 